\title{
Developments and open problems on chiral polytopes
}

\author{
Daniel Pellicer \\ National University of Mexico, Morelia, Michoacan, Mexico
}

Received 30 December 2010, accepted 28 January 2012, published online 30 May 2012

\begin{abstract}
This paper presents the current state of the theory of abstract chiral polytopes together with 39 open problems on this topic.
\end{abstract}

Keywords: Chiral polytope, regular and chiral.

Math. Subj. Class.: 05C25, 51M20

\section{Introduction}

Abstract polytopes are combinatorial structures that generalize the concept of convex polytopes. Although some ideas can be found earlier [32], the work of Coxeter and Grünbaum in the 1970's inspired later work such as [37] and it mainly deals with those polytopes with a high degree of symmetry.

Regular abstract polytopes are those admitting all symmetries by reflections, which generate all other possible symmetries. Most results regarding regular abstract polytopes are summarized in [37].

Generally, an object is said to be chiral whenever it lacks mirror symmetries. In principle it may have no symmetries at all. In the 1980's, in [57] the term "chiral polytopes" was reserved for those abstract polytopes admitting all possible symmetries by abstract rotations but no mirror symmetries. In some way, "chiral polytopes" became a short name for "the most symmetric chiral polytopes". In this paper we shall be consistent with the work done so far and use the term "chiral" exclusively for polytopes admitting all rotational symmetries, although we acknowledge the existence of other interesting classes of polytopes with no mirror symmetries.

After regular polytopes, chiral polytopes are regarded as the most symmetric ones. However, there is a considerable difference in the knowledge we have about these two classes of polytopes. This is due in part to the distinct properties of regular and chiral polytopes that make chirality harder to study. For instance, in contrast with regular polytopes,

E-mail address: pellicer@matmor.unam.mx (Daniel Pellicer) 
there are no convex chiral polytopes, and we do not know of a natural family of chiral polytopes providing an example in each rank. Consequently, several questions that have been answered for regular polytopes remain open for chiral polytopes. Part of the goal of this paper is to provide a number of properties with respect to which regular and chiral polytopes behave in different ways. We also state a number of open problems dealing with chiral polytopes.

In Section 2 we state the main definitions and give a brief exposition of properties of regular and chiral polytopes. From Section 3 to Section 8 we discuss group actions, Petrie paths, topological considerations, maps and polyhedra, higher ranks and realizations, all in relation with regular and chiral polytopes.

\section{Definitions}

We start by introducing abstract regular and chiral polytopes, referring to [37], [57] for details.

An abstract d-polytope (or abstract polytope of rank $d$ ) $\mathcal{K}$ is a partially ordered set satisfying the following properties. The elements of $\mathcal{K}$ are called faces, and its maximal totally ordered subsets are called flags. There is a minimum face $F_{-1}$ and a maximum face $F_{d}$, and every flag of $\mathcal{K}$ contains precisely $d+2$ elements (including $F_{-1}$ and $F_{d}$ ). This induces a rank function from $\mathcal{K}$ to the set $\{-1,0, \ldots, d\}$ such that $\operatorname{rank}\left(F_{-1}\right)=-1$ and $\operatorname{rank}\left(F_{d}\right)=d$. The faces of rank $i$ are called $i$-faces, the 0 -faces are called vertices, the 1 -faces are called edges and the $(d-1)$-faces are called facets. When convenient we shall abuse notation and identify the section $G / F_{-1}:=\{H \mid H \leq G\}$ with the face $G$ itself in $\mathcal{K}$. Given a vertex $v$, the section $F_{d} / v:=\{H \mid H \geq v\}$ is called the vertex-figure of $\mathcal{K}$ at $G$. For every incident faces $F$ and $G$ such that $\operatorname{rank}(F)-\operatorname{rank}(G)=2$, there exist precisely two faces $H_{1}$ and $H_{2}$ such that $G<H_{1}, H_{2}<F$. This property is referred to as the diamond condition. The diamond condition implies that for any flag $\Phi$ and any $i \in$ $\{0, \ldots, d-1\}$ there exists a unique flag $\Phi^{i}$ that differs from $\Phi$ only in the $i$-face. This flag is called the $i$-adjacent flag of $\Phi$. Finally, $\mathcal{K}$ must be strongly flag-connected, in the sense that for any two flags $\Phi, \Phi^{\prime}$ there exists a sequence of flags $\Psi_{0}=\Phi, \Psi_{1}, \ldots, \Psi_{m}=\Phi^{\prime}$ such that $\Phi \cap \Phi^{\prime} \subseteq \Psi_{k}$ and $\Psi_{k-1}$ is adjacent to $\Psi_{k}$, for $k=1, \ldots, m$.

Whenever the (polygonal) section $G / F:=\{H \mid F \leq H \leq G\}$ between an $(i-2)$-face $F$ and an incident $(i+1)$-face $G$ of an abstract polytope depends only on $i$ and not on $F$ and $G$, we say that $\mathcal{K}$ is equivelar. If $F$ and $G$ are faces, with ranks $i-2$ and $i+1$ respectively, of an equivelar polytope $\mathcal{K}$, the section $G / F$ is isomorphic to an abstract $p_{i}$-gon where $p_{i} \leq \infty$ is fixed. We define the Schläfli type of an equivelar polytope $\mathcal{K}$ as $\left\{p_{1}, \ldots, p_{d-1}\right\}$. Throughout this paper we will encounter only polytopes with $p_{i} \geq 3$ for $i=1, \ldots, d-1$. Regular and chiral polytopes defined below are examples of equivelar polytopes.

An automorphism of a polytope $\mathcal{K}$ is an order-preserving permutation of its faces. The automorphisms of $\mathcal{K}$ form a group denoted by $\Gamma(\mathcal{K})$.

We say that a $d$-polytope $\mathcal{K}$ is regular whenever $\Gamma(\mathcal{K})$ acts transitively on the flags of $\mathcal{K}$. In this case, $\Gamma(\mathcal{K})$ is generated by involutions $\rho_{0}, \ldots, \rho_{d-1}$ where $\rho_{i}$ is the unique automorphism of $\mathcal{K}$ mapping a fixed base flag $\Phi$ to its $i$-adjacent flag $\Phi^{i}$. The generating set $\left\{\rho_{0}, \ldots, \rho_{d-1}\right\}$ satisfies the relations

$$
\begin{aligned}
\rho_{i}^{2} & =\varepsilon \quad \text { for all } i \\
\left(\rho_{i} \rho_{j}\right)^{2} & =\varepsilon \quad \text { whenever }|i-j| \geq 2,
\end{aligned}
$$


where $\varepsilon$ denotes the identity element. These generators also satisfy the intersection conditions given by

$$
\left\langle\rho_{i} \mid i \in I\right\rangle \cap\left\langle\rho_{i} \mid i \in J\right\rangle=\left\langle\rho_{i} \mid i \in I \cap J\right\rangle,
$$

for all $I, J \subseteq\{0, \ldots, d-1\}$. The order of the element $\rho_{i-1} \rho_{i}$ in $\Gamma(\mathcal{K})$ is the Schläfli number $p_{i}$. In this paper $p_{i}$ may be either finite or $\infty$.

A group together with a generating set $\left\{\rho_{0}, \ldots, \rho_{d-1}\right\}$ is called a string $C$-group if it satisfies (2.1) and (2.2), and the intersection condition (2.3). String C-groups are in a oneto-one correspondence with automorphism groups of regular polytopes; in particular, every regular polytope can be reconstructed from its automorphism group and its distinguished generators.

The rotation subgroup of the automorphism group of a regular $d$-polytope $\mathcal{K}$ is defined as the subgroup of $\Gamma(\mathcal{K})$ consisting of words of even length on the generators $\rho_{0}, \ldots, \rho_{d-1}$ and is denoted by $\Gamma^{+}(\mathcal{K})$. For convenience, the rotation subgroup of $\Gamma(\mathcal{K})$ is simply referred to as the rotation subgroup of $\mathcal{K}$. For $i=1, \ldots, d-1$ we define the abstract rotation $\sigma_{i}$ to be $\rho_{i-1} \rho_{i}$, that is, the automorphism of $\mathcal{K}$ mapping the base flag $\Phi$ to $\left(\Phi^{i}\right)^{i-1}$. It is not hard to see that $\Gamma^{+}(\mathcal{K})=\left\langle\sigma_{1}, \ldots, \sigma_{d-1}\right\rangle$ and that those generators satisfy the relations

$$
\left(\sigma_{i} \sigma_{i+1} \cdots \sigma_{j}\right)^{2}=\varepsilon
$$

for $i<j$. In particular, $\sigma_{i} \sigma_{i+1} \cdots \sigma_{j}=\sigma_{j}^{-1} \cdots \sigma_{i+1} \sigma_{i}^{-1}$ for $j \geq i+1$. The order of $\sigma_{i}$ is precisely the entry $p_{i}$ in the Schläfli symbol.

We define abstract half-turns as the involutions $\tau_{i, j}:=\sigma_{i} \cdots \sigma_{j}$ for $i<j$. For consistency we also define $\tau_{0, i}:=\tau_{i, d}:=\varepsilon$ and denote $\sigma_{i}$ by $\tau_{i, i}$. Then the abstract rotations and half-turns satisfy the intersection condition given by

$$
\left\langle\tau_{i, j} \mid i \leq j ; i-1, j \in I\right\rangle \cap\left\langle\tau_{i, j} \mid i \leq j ; i-1, j \in J\right\rangle=\left\langle\tau_{i, j} \mid i \leq j ; i-1, j \in I \cap J\right\rangle
$$

for $I, J \subseteq\{-1, \ldots, d\}$.

For any regular polytope $\mathcal{K}$, the index of $\Gamma^{+}(\mathcal{K})$ in $\Gamma(\mathcal{K})$ is at most 2. Whenever $\Gamma^{+}(\mathcal{K})$ has index 2 in $\Gamma(\mathcal{K})$ we say that $\mathcal{K}$ is orientably regular; otherwise $\mathcal{K}$ is said to be non-orientably regular.

We say that the $d$-polytope $\mathcal{K}$ is chiral whenever its automorphism group induces two orbits on flags with the property that adjacent flags belong to different orbits. This eliminates all possible abstract reflections.

Note that every polygon is isomorphic (as a poset) to a regular convex polygon. Therefore there are no chiral 2-polytopes.

Although the facets and vertex-figures of a chiral $d$-polytope $\mathcal{K}$ can be either regular or chiral, the 2 -faces and $(d-2)$-faces of $\mathcal{K}$ must necessarily be regular (see [57, Proposition 9]).

Some quotients of the Euclidean tessellations $\{3,6\},\{4,4\}$ and $\{6,3\}$ of the Euclidean plane by triangles, squares and hexagons respectively are examples of orientably regular and chiral polyhedra (rank 3 polytopes). Let $T$ denote the group of translations of the plane which fix a given tessellation. We denote by $\{p, q\}_{\bar{u}}$ the quotient of the regular tessellation $\{p, q\}$ of the plane by the subgroup $T_{\bar{u}}$ of $T$ generated by the translations with respect to the linearly independent vectors $\bar{u}$ and $\bar{u} R$, where $R$ stands for the rotation by $2 \pi / q$. For $\{p, q\}=\{4,4\}$ and $\{p, q\}=\{3,6\}$ it is well known that the quotient $\{p, q\}_{\bar{u}}$ is regular if and only if $\bar{u}=(a, 0)$ or $\bar{u}=(a, a)$ with the vectors taken with respect to the basis $\{e, e R\}$ where $e$ denotes a vector with the size and direction of an edge of the tessellation. 
Moreover, the quotient $\{p, q\}_{\bar{u}}$ is chiral whenever it is not regular (see [19], [37, Section 1D]). The same is true for $\{p, q\}=\{6,3\}$ if we choose $e$ as the vector between the centres of two hexagons sharing an edge. Note that for $a=0,1$ the maps $\{4,4\}_{(1, a)},\{3,6\}_{(1,0)}$ and $\{6,3\}_{(1,0)}$ fail to be strongly flag-connected, and thus, they are not abstract polyhedra.

The automorphism group $\Gamma(\mathcal{K})$ of a chiral polytope is generated by elements $\sigma_{1}, \ldots$, $\sigma_{d-1}$, where $\sigma_{i}$ maps a base flag $\Phi$ to $\left(\Phi^{i}\right)^{i-1}$, in which case, $\sigma_{i}$ cyclically permutes the $i$ and $(i-1)$-faces of $\mathcal{K}$ incident with the $(i-2)$ - and $(i+1)$-faces of $\Phi$. Furthermore, the generators $\sigma_{i}$ also satisfy (2.4) as well as the intersection conditions in (2.5). Because of the obvious similarities between the automorphism groups of chiral polytopes and the rotation subgroups of regular polytopes we shall also refer to the generators $\sigma_{i}$ of the automorphism groups of chiral polytopes as abstract rotations. The products $\tau_{i, j}:=\sigma_{i} \sigma_{i+1} \cdots \sigma_{j}(i<j)$ will be referred to as abstract half-turns. Furthermore, we say that the rotation subgroup of a chiral polytope is its automorphism group, and when convenient, we shall denote the automorphism group $\Gamma(\mathcal{K})=\left\langle\sigma_{1}, \ldots, \sigma_{d-1}\right\rangle$ of the chiral polytope $\mathcal{K}$ by $\Gamma^{+}(\mathcal{K})$.

It was proved in [57] that any group $\Gamma$ together with a generating set $\left\{\sigma_{1}, \ldots, \sigma_{d-1}\right\}$ satisfying (2.4) and the intersection conditions in (2.5) is the rotation subgroup of either an orientably regular polytope, or a chiral polytope. Furthermore, $\Gamma$ is the rotation subgroup of a regular polytope if and only if there is a group automorphism $\eta$ of $\Gamma$ such that

$$
\sigma_{i} \eta= \begin{cases}\sigma_{i}^{-1} & \text { for } i=1 \\ \sigma_{1}^{2} \sigma_{i} & \text { for } i=2 \\ \sigma_{i} & \text { for } i>2\end{cases}
$$

The following lemmas provide some properties of the generators of the automorphism groups of chiral polytopes.

Lemma 2.1. Let $\Gamma(\mathcal{K})=\left\langle\sigma_{1}, \ldots, \sigma_{d-1}\right\rangle$ be the automorphism group of a chiral $d$-polytope, then $\sigma_{i} \sigma_{j}=\sigma_{j} \sigma_{i}$ whenever $|i-j| \geq 3$.

Proof. Without loss of generality assume that $i<j$. We use (2.4) to prove that $\sigma_{i}$ commutes with $\sigma_{j}^{-1}$.

$$
\begin{aligned}
\sigma_{i} \sigma_{j}^{-1} & =\sigma_{i}\left(\sigma_{i+1} \sigma_{i+2} \cdots \sigma_{j} \sigma_{i+1} \sigma_{i+2} \cdots \sigma_{j-1}\right) \\
& =\left(\sigma_{i} \sigma_{i+1} \cdots \sigma_{j}\right) \sigma_{i+1} \sigma_{i+2} \cdots \sigma_{j-1} \\
& =\left(\sigma_{j}^{-1} \sigma_{j-1}^{-1} \cdots \sigma_{i}^{-1}\right) \sigma_{i+1} \sigma_{i+2} \cdots \sigma_{j-1} \\
& =\sigma_{j}^{-1}\left(\sigma_{j-1}^{-1} \sigma_{j-2}^{-1} \cdots \sigma_{i}^{-1}\right) \sigma_{i+1} \sigma_{i+2} \cdots \sigma_{j-1} \\
& =\sigma_{j}^{-1}\left(\sigma_{i} \sigma_{i+1} \cdots \sigma_{j-1}\right) \sigma_{i+1} \sigma_{i+2} \cdots \sigma_{j-1} \\
& =\sigma_{j}^{-1} \sigma_{i}\left(\sigma_{i+1} \sigma_{i+2} \cdots \sigma_{j-1}\right)^{2}=\sigma_{j}^{-1} \sigma_{i} .
\end{aligned}
$$

Lemma 2.2. Let $\Gamma(\mathcal{K})=\left\langle\sigma_{1}, \ldots, \sigma_{d-1}\right\rangle$ be the automorphism group of a chiral $d$-polytope and $\tau$ the product $\sigma_{1} \sigma_{2} \cdots \sigma_{d-1}$. Then

$$
\text { 1. } \tau \sigma_{i} \tau= \begin{cases}\sigma_{i}^{-1} & \text { for } \quad i=1, d-1 \\ \sigma_{i} & \text { for } \quad i=3,4, \ldots, d-3\end{cases}
$$


2. $\sigma_{2 i}^{-1} \sigma_{2 j+1} \sigma_{2 i}=\left\{\begin{array}{lll}\sigma_{2 i}^{-2} \sigma_{2 j+1}^{-1} & \text { if } & j=i-1, i \\ \sigma_{2 j+1} & \text { if } & j \neq i-1, i\end{array}\right.$

Proof. We use (2.4) and Lemma 2.1.

$$
\begin{aligned}
\tau \sigma_{1} \tau & =\sigma_{1} \sigma_{2} \cdots \sigma_{d-1}\left(\sigma_{1}^{2}\right) \sigma_{2} \sigma_{3} \cdots \sigma_{d-1}=\sigma_{1} \sigma_{2} \sigma_{3}\left(\sigma_{1}^{2}\right) \sigma_{4} \sigma_{5} \cdots \sigma_{d-1} \sigma_{2} \sigma_{3} \cdots \sigma_{d-1} \\
& =\sigma_{1} \sigma_{2} \sigma_{3} \sigma_{1}^{2}\left(\sigma_{4} \sigma_{5} \cdots \sigma_{d-1} \sigma_{2} \sigma_{3} \cdots \sigma_{d-1}\right)=\left(\sigma_{1} \sigma_{2} \sigma_{3}\right) \sigma_{1}^{2}\left(\sigma_{3}^{-1} \sigma_{2}^{-1}\right) \\
& =\left(\sigma_{3}^{-1} \sigma_{2}^{-1} \sigma_{1}^{-1}\right) \sigma_{1}^{2} \sigma_{3}^{-1} \sigma_{2}^{-1}=\left(\sigma_{3}^{-1} \sigma_{2}^{-1}\right) \sigma_{1}\left(\sigma_{3}^{-1} \sigma_{2}^{-1}\right)=\left(\sigma_{2} \sigma_{3}\right) \sigma_{1}\left(\sigma_{2} \sigma_{3}\right) \\
& =\sigma_{1}^{-1}
\end{aligned}
$$

The proof of $\tau \sigma_{d-1} \tau=\sigma_{d-1}^{-1}$ is analogous.

On the other hand, for $4 \leq i \leq d-4$,

$$
\begin{aligned}
\tau \sigma_{i} \tau= & \sigma_{1} \sigma_{2} \cdots \sigma_{d-1}\left(\sigma_{i}\right) \sigma_{1} \sigma_{2} \cdots \sigma_{d-1}=\sigma_{1} \sigma_{2} \cdots \sigma_{d-1} \sigma_{1} \sigma_{2} \cdots \\
& \cdots \sigma_{i-3}\left(\sigma_{i}\right) \sigma_{i-2} \sigma_{i-1} \cdots \sigma_{d-1} \\
= & \left(\sigma_{1} \sigma_{2} \cdots \sigma_{d-1}\right) \sigma_{1} \sigma_{2} \cdots \sigma_{i-3} \sigma_{i} \sigma_{i-2} \sigma_{i-1} \cdots \sigma_{d-1} \\
= & \left(\sigma_{d-1}^{-1} \sigma_{d-2}^{-1} \cdots \sigma_{1}^{-1}\right) \sigma_{1} \cdots \sigma_{i-3} \sigma_{i}\left(\sigma_{i-2} \sigma_{i-1} \cdots \sigma_{d-1}\right) \\
= & \sigma_{d-1}^{-1} \sigma_{d-2}^{-1} \cdots \sigma_{i-2}^{-1} \sigma_{i}\left(\sigma_{d-1}^{-1} \sigma_{d-2}^{-1} \cdots \sigma_{i-2}^{-1}\right) \\
= & \left(\sigma_{d-1}^{-1} \sigma_{d-2}^{-1} \cdots \sigma_{i-2}^{-1}\right)\left(\sigma_{i}\right) \sigma_{d-1}^{-1} \sigma_{d-2}^{-1} \cdots \sigma_{i-2}^{-1} \\
= & \left(\sigma_{i-2} \cdots \sigma_{d-1}\right) \sigma_{d-1}^{-1} \sigma_{d-2}^{-1} \cdots \sigma_{i+3}^{-1}\left(\sigma_{i}\right) \sigma_{i+2}^{-1} \sigma_{i+1}^{-1} \cdots \sigma_{i-2}^{-1} \\
= & \sigma_{i-2} \sigma_{i-1}\left(\sigma_{i} \sigma_{i+1} \sigma_{i+2} \sigma_{i}\right) \sigma_{i+2}^{-1} \sigma_{i+1}^{-1}\left(\sigma_{i}^{-1} \sigma_{i-1}^{-1} \sigma_{i-2}^{-1}\right) \\
= & \sigma_{i-2} \sigma_{i-1}\left(\sigma_{i+2}^{-1} \sigma_{i+1}^{-1}\right) \sigma_{i+2}^{-1} \sigma_{i+1}^{-1}\left(\sigma_{i-2} \sigma_{i-1} \sigma_{i}\right) \\
= & \sigma_{i-2} \sigma_{i-1}\left[\left(\sigma_{i+1} \sigma_{i+2}\right)^{2}\right]^{-1} \sigma_{i-2} \sigma_{i-1} \sigma_{i}=\sigma_{i-2} \sigma_{i-1} \sigma_{i-2} \sigma_{i-1} \sigma_{i}=\sigma_{i}
\end{aligned}
$$

The same procedure, but with less steps, can be used for $i \in\{3, d-3\}$. This proves the first part.

The second part is a direct consequence of Lemma 2.1 and (2.4).

Lemma 2.3. Let $\Gamma(\mathcal{K})=\left\langle\sigma_{1}, \ldots, \sigma_{d-1}\right\rangle$ be the automorphism group of a chiral $d$-polytope. Then

$$
\sigma_{1} \sigma_{2} \cdots \sigma_{k} \sigma_{k+2}=\sigma_{k+1} \sigma_{1} \sigma_{2} \cdots \sigma_{k+2}
$$

for all $k \geq 2$.

Proof. Note that (2.4) implies that $\sigma_{k+1} \sigma_{k-1} \sigma_{k} \sigma_{k+1}=\sigma_{k}^{-1} \sigma_{k-1}^{-1}=\sigma_{k-1} \sigma_{k}$. The lemma then follows from the commutativity of $\sigma_{k+1}$ with $\sigma_{i}$ for $i<k-1$.

Lemma 2.4. Let $\Gamma(\mathcal{K})=\left\langle\sigma_{1}, \ldots, \sigma_{d-1}\right\rangle$ be the automorphism group of a chiral $d$-polytope. Then

1. $\sigma_{1}^{-1} \sigma_{3} \sigma_{5} \cdots \sigma_{2 k+1}=\sigma_{2} \sigma_{4} \cdots \sigma_{2 k} \sigma_{1} \sigma_{2} \cdots \sigma_{2 k+1}$,

2. $\sigma_{1} \sigma_{2} \sigma_{4} \sigma_{6} \cdots \sigma_{2 k}=\sigma_{3} \sigma_{5} \cdots \sigma_{2 k-1} \sigma_{1} \sigma_{2} \cdots \sigma_{2 k}$.

Proof. We proceed by induction over $k$. Since $\left(\sigma_{1} \sigma_{2}\right)^{2}=\varepsilon$ it follows that $\sigma_{1}^{-1} \sigma_{3}=$ $\sigma_{2} \sigma_{1} \sigma_{2} \sigma_{3}$. On the other hand, $\sigma_{1} \sigma_{2} \sigma_{4}=\sigma_{3} \sigma_{1} \sigma_{2} \sigma_{3} \sigma_{4}$ is a particular instance of Lemma 2.3 .

The inductive step follows directly from Lemma 2.3. 
Each chiral $d$-polytope $\mathcal{K}$ occurs in two isomorphic enantiomorphic forms, in a sense in a right and left handed version which can be thought of as mirror images of each other. The two enantiomorphic forms correspond to the two possible choices of flag orbit for the base flag. The polyhedra $\{4,4\}_{(2,1)}$ and $\{4,4\}_{(1,2)}$ constitute an example of the two enantiomorphic forms of a chiral polytope. The group $\Gamma(\mathcal{K})$ together with the distinguished generators $\sigma_{1}, \ldots, \sigma_{d-1}$ completely determines the chiral polytope $\mathcal{K}$ as well as the enantiomorphic form in consideration. The group of the other enantiomorphic form is also $\Gamma(\mathcal{K})$, but with respect to the generators $\sigma_{1}^{-1}, \sigma_{1}^{2} \sigma_{2}, \sigma_{3}, \ldots, \sigma_{d-1}$. We refer to [58] for details.

The $d$ ual $\mathcal{K}^{*}$ of a $d$-polytope $\mathcal{K}$ is the $d$-polytope consisting of the set of faces of $\mathcal{K}$ with the order reversed. Note that the dual of a regular (resp. chiral) polytope is again regular (resp. chiral).

\section{Group structures}

There are several groups associated with chiral abstract polytopes. The most obvious one is the automorphism group of the polytope itself.

Not every group is the automorphism group of a chiral polytope. For instance, abelian groups cannot be generated by two or more elements $\sigma_{i}$ of order at least 3 satisfying (2.4).

In recent years there have been attempts to describe all finite simple groups in terms of their actions on particular sets (see for example [31] and [30]). This gives interest to the problem of determining which simple groups are automorphism groups of chiral $d$ polytopes. Alternating groups and projective special linear groups were first investigated in [46] and [58] respectively.

Problem 1 Determine which finite simple groups are automorphism groups of chiral $d$-polytopes.

Of particular interest are the sporadic simple groups.

Problem 2 Determine which sporadic finite simple groups are automorphism groups of chiral $d$-polytopes.

For any $d$-polytope $\mathcal{K}$ (not necessarily regular or chiral) and $i=1, \ldots, d-1$ we define the (involutory) permutation $r_{i}$ on the flags of $\mathcal{K}$ by $\Phi r_{i}=\Phi^{i}$. The subgroup $\left\langle r_{0}, \ldots, r_{d-1}\right\rangle$ of the symmetric group on the set of flags of $\mathcal{K}$ is often referred to as the monodromy group of $\mathcal{K}$ (see for example [27], [29]).

Whenever the polytope $\mathcal{K}$ is regular, its monodromy group is isomorphic to its automorphism group. For less symmetric polytopes this is no longer the case. If $\mathcal{K}$ is chiral then there is a contravariant group isomorphism between its automorphism group with respect to the base flag $\Phi$ and the permutation group determined by the action on the flag orbit containing $\Phi$ of the set of even words on the generators $r_{0}, \ldots, r_{d-1}$ of the monodromy group (see [49, Proposition 2.7]).

Let $\mathcal{K}$ be a $d$-polytope and $\mathcal{P}$ be a regular $d$-polytope with automorphism group $\Gamma(\mathcal{P})=$ $\left\langle\rho_{0}, \ldots, \rho_{d-1}\right\rangle$. We say that $\mathcal{K}$ admits a flag action from $\mathcal{P}$ if there is a group homomorphism from $\Gamma(\mathcal{P})$ to the monodromy group of $\mathcal{K}$ mapping $\rho_{i}$ to $r_{i}$ for all $i$. The polytope $\mathcal{K}$ is isomorphic to the quotient $\mathcal{P} / N$ where $N$ is the stabilizer in $\Gamma(\mathcal{P})$ of a flag of $\mathcal{K}$. Here $\mathcal{P} / N$ can be thought of as the combinatorial structure resulting from identifying the image $\Phi w$ of the base flag $\Phi$ with all flags of the form $\Phi h w$ where $h \in N$, for every $w$ in the monodromy group. We refer to [22] and [37, Section 2D] for more information on flag actions and quotients. 
We say that the regular polytope $\mathcal{P}$ is a regular cover of the polytope $\mathcal{K}$ whenever $\mathcal{K}$ admits a flag action from $\mathcal{P}$. Moreover, $\mathcal{P}$ is the minimal regular cover of $\mathcal{K}$ if it admits a flag action from all other regular covers of $\mathcal{K}$. It is not hard to see that whenever the generators $r_{0}, \ldots, r_{d-1}$ of the monodromy group of $\mathcal{K}$ satisfy the intersection condition (2.3) then $\operatorname{Mon}(\mathcal{K})$ is the automorphism group of the minimal regular cover of $\mathcal{K}$. Further details can be found in [26] and [41].

It is known that the monodromy group of all polyhedra are string C-groups [41], and therefore every polyhedron has a minimal regular cover. This is not the case for higher rank polytopes. In [40] an example of a 4-polytope whose monodromy group is not a string C-group is given.

The mix of two regular polytopes is defined in [37, Section 7A]. This definition is extended to the mix of orientably regular or chiral polytopes in [5] and [49] as follows.

Let $\Gamma^{+}(\mathcal{K})$ and $\Gamma^{+}(\mathcal{P})$ be the rotation subgroups of orientably regular or chiral $d$ polytopes with abstract rotations given by $\sigma_{1}, \ldots \sigma_{d-1}$ and $\delta_{1}, \ldots, \delta_{d-1}$ respectively. The $\operatorname{mix} \Gamma^{+}(\mathcal{K}) \diamond \Gamma^{+}(\mathcal{P})$ of $\Gamma^{+}(\mathcal{K})$ and $\Gamma^{+}(\mathcal{P})$ is the subgroup $\left\langle\left(\sigma_{1}, \delta_{1}\right), \ldots,\left(\sigma_{d-1}, \delta_{d-1}\right)\right\rangle$ of the direct product $\Gamma^{+}(\mathcal{K}) \times \Gamma^{+}(\mathcal{P})$. Whenever $\Gamma^{+}(\mathcal{K}) \diamond \Gamma^{+}(\mathcal{P})$ satisfies the intersection condition with respect to the generators $\gamma_{i}:=\left(\sigma_{i}, \delta_{i}\right)$, the group $\Gamma^{+}(\mathcal{K}) \diamond \Gamma^{+}(\mathcal{P})$ is the rotation subgroup of an orientably regular or chiral polytope $\mathcal{K} \diamond \mathcal{P}$ called the mix of $\mathcal{K}$ and $\mathcal{P}$.

The monodromy group of a chiral polytope $\mathcal{K}$ is the mix of $\mathcal{K}$ with its enantiomorphic form. The resulting group is a string $\mathrm{C}$-group whenever the facets or vertex-figures of $\mathcal{K}$ are regular. The corresponding minimal regular cover is called mixed regular cover in [49, Section 4]. However it is not known whether all chiral polytopes with chiral facets and chiral vertex-figures have a minimal regular cover.

Problem 3 Is there a chiral polytope whose monodromy group is not a string C-group?

Even if the previous problem has a negative answer, it is still interesting to determine whether this implies that some chiral polytopes have no minimal regular cover.

Problem 4 Does every chiral polytope have a minimal regular cover?

It is easy to see that whenever $\mathcal{K}$ and $\mathcal{P}$ are regular and $\mathcal{K} \diamond \mathcal{P}$ is a polytope, the latter is the minimal regular polytope which covers simultaneously $\mathcal{K}$ and $\mathcal{P}$. An equivalent statement for the case when either $\mathcal{K}$ or $\mathcal{P}$ is chiral must hold for an appropriate definition of a quotient of a chiral polytope.

Problem 5 Develop a theory of chiral covers of polytopes.

Any $d$-polytope $\mathcal{K}$ is a quotient of the universal polytope $\left\{\infty^{d-1}\right\}:=\{\infty, \ldots, \infty\}$ with the Coxeter group

$$
\left.\left[\infty^{d-1}\right]=\left\langle\rho_{0}, \ldots, \rho_{d-1}\right|\left(\rho_{i} \rho_{j}\right)^{2}=i d \text { for }|i-j| \geq 2\right\rangle
$$

as automorphism group. Let $M$ be the subgroup of $\left[\infty^{d-1}\right]$ such that $\mathcal{K}=\left\{\infty^{k-1}\right\} / M$. The subgroup $M$ is normal in $\left[\infty^{d-1}\right]$ if and only if $\mathcal{K}$ is regular. If $\mathcal{K}$ is chiral, then $M$ is normal in the subgroup $\left[\infty^{k-1}\right]^{+}$consisting of the elements of $\left[\infty^{k-1}\right]$ that can be expressed as a word of even length in the generators. The chirality group of a chiral polytope $\mathcal{K}$ is defined in [5] as $X(\mathcal{K}):=M /\left(M \cap \rho_{0} M \rho_{0}\right)$, or equivalently as $X(\mathcal{K})=$ $\left(M \cdot \rho_{0} M \rho_{0}\right) / M$. Intuitively, the order of $X(\mathcal{K})$ is a measure of how far from regular 
(of how chiral) is $\mathcal{K}$. This concept preserves the idea of the chirality group of hypermaps defined in [4].

The chirality group $X(\mathcal{K} \diamond \mathcal{P})$ of the mix of a chiral polytope $\mathcal{K}$ and a directly regular polytope $\mathcal{P}$ is shown in [5] to be a subgroup of $X(\mathcal{K})$. These two groups sometimes coincide.

Problem 6 Can we characterize all pairs $(\mathcal{K}, \mathcal{P})$ consisting of a chiral polytope $\mathcal{K}$ and a regular polytope $\mathcal{P}$ such that $X(\mathcal{K} \diamond \mathcal{P})=X(\mathcal{K})$ ?

\section{Petrie paths}

A Petrie path $\pi$ of a $d$-polytope $\mathcal{K}$ with rank at least 3 is a closed or infinite walk on the underlying graph of $\mathcal{K}$ (the set of vertices and edges) such that for $2 \leq k \leq d-1$, every $k$ consecutive edges of $\pi$ belong to the same $k$-face, but the $(k+1)$-th does not belong to the common $k$-face of the previous $k$ edges. An equivalent recursive definition of Petrie paths was given in [18, Chapter 12.4].

According to [65], a Petrie map $\Pi(\xi)$ of a $d$-polytope $\mathcal{K}$ is the (left) action of the product $r_{\xi(0)} r_{\xi(1)} \cdots r_{\xi(d-1)}$ of the generators $r_{0}, \ldots, r_{d-1}$ of the monodromy group of $\mathcal{K}$, for a given permutation $\xi$ of $\{0,1, \ldots, d-1\}$. A Petrie scheme of $\mathcal{K}$ is a sequence of flags $\left(\Phi, \Pi \Phi, \ldots, \Pi^{m-1} \Phi\right)$, where $\Phi$ is a flag of $\mathcal{K}, \Pi=\Pi(\xi)$ is a Petrie map, $\Pi^{m} \Phi=\Phi$ and $\Pi^{k} \Phi \neq \Phi$ for $1 \leq k \leq m-1$; or the sequence $\left(. ., \Pi^{-1} \Phi, \Phi, \Pi \Phi, \Pi^{2} \Phi, \ldots\right)$ whenever $\Pi^{k} \Phi \neq \Phi$ for all $k \neq 0$.

A Petrie scheme $P$ of a polytope $\mathcal{K}$ is said to be acoptic whenever each proper face of $\mathcal{K}$ is contained in at most one flag of $P$ ([26, Section 6]). Several families of polytopes were proved to be acoptic in [65].

Problem 7 Determine conditions under which the Petrie scheme of a chiral polytope is acoptic.

The following theorem is equivalent to Theorem 2.5 in [65].

Theorem 4.1. Any Petrie map of a $d$-polytope with monodromy group $\left\langle r_{0}, \ldots, r_{d-1}\right\rangle$ is a conjugate of the Petrie map $\Pi(\iota)$ where $\iota$ is the identity permutation of $\{0, \ldots, d-1\}$.

The Petrie schemes associated to the Petrie map $\Pi(\xi)$ of a regular $d$-polytope $\mathcal{K}$ are precisely the sequences $\left(\Phi, \psi \Phi, \ldots, \psi^{m-1} \Phi\right)$ or $\left(\ldots, \psi^{-1} \Phi, \Phi, \psi \Phi, \psi^{2} \Phi, \ldots\right)$ where $\psi:=$ $\rho_{\xi(d-1)} \rho_{\xi(d-2)} \cdots \rho_{\xi(0)} \in \Gamma(\mathcal{K})$, since the actions of $\Pi(\xi)$ and $\psi$ on any flag coincide. It follows from Theorem 4.1 that all Petrie paths are equivalent under $\Gamma(\mathcal{K})$.

Whenever $\mathcal{K}$ is a chiral $d$-polytope with $d$ even, the Petrie map $\Pi(\xi)$ acts on the base flag like an automorphism of $\mathcal{K}$. In particular, $r_{d-1} r_{d-2} \cdots r_{0}$ acts like $\sigma_{1} \sigma_{3} \cdots \sigma_{d-1}$. Theorem 4.1 and the contravariant isomorphism of $\Gamma(\mathcal{K})$ to a permutation group on the orbit of the base flag (see Section 3) imply that there are precisely two classes of Petrie schemes under $\Gamma(\mathcal{K})$, namely the one induced by the product $r_{d-1} r_{d-2} \cdots r_{0}$ (or by the automorphism $\sigma_{1} \sigma_{3} \cdots \sigma_{d-1}$ ) and the one induced by the product $r_{d-1} r_{d-2} \cdots r_{2} r_{0} r_{1}$ (or by the automorphism $\sigma_{1}^{-1} \sigma_{3} \cdots \sigma_{d-1}$ ).

If $\mathcal{K}$ is a chiral $d$-polytope with $d$ odd, then the numbers of factors of the Petrie maps are odd, and therefore all Petrie schemes must involve an even number of Petrie maps, when there are finitely many. That is, for any odd number $k$ and any Petrie map $\Pi=$ $\Pi(\xi), \Pi^{k} \Phi \neq \Phi$. The square of a Petrie map acts on the base flag like an automorphism of $\mathcal{K}$. For instance, if $\Pi$ is the action of the product $r_{i-1} r_{i-2} r_{i-3} \cdots r_{0}$ then $\Pi^{2}$ 
acts like $\sigma_{1} \sigma_{3} \cdots \sigma_{d-2} \sigma_{1} \sigma_{2} \cdots \sigma_{d-1} \sigma_{2} \sigma_{4} \cdots \sigma_{d-1}$. As above we can conclude that there are precisely two classes of Petrie schemes under $\Gamma(\mathcal{K})$, the one induced by the product $r_{d-1} r_{d-2} \cdots r_{0}$ (or by the automorphism $\sigma_{1} \sigma_{3} \cdots \sigma_{d-2} \sigma_{1} \sigma_{2} \cdots \sigma_{d-1} \sigma_{2} \sigma_{4} \cdots \sigma_{d-1}$ ) and the one induced by the product $r_{d-1} r_{d-2} \cdots r_{2} r_{0} r_{1}$ (or by the automorphism $\sigma_{1}^{-1} \sigma_{3} \cdots$ $\left.\sigma_{d-2} \sigma_{1} \sigma_{2} \cdots \sigma_{d-1} \sigma_{1}^{2} \sigma_{2} \sigma_{4} \cdots \sigma_{d-1}\right)$.

An inductive argument shows that for every $d-1 \geq k>j \geq 2$, the $j$-face of $r_{k-1} r_{k-2} \cdots r_{0}\left(r_{d-1} r_{d-2} \cdots r_{0}\right)^{d-k-1} \Phi$ is contained in the $k$-face of $\Phi$. It follows that the set of vertices and edges of the flags of a Petrie scheme determine a Petrie path of the polytope. Moreover, the Petrie paths of a polytope coincide with the set of vertices and edges of its Petrie schemes.

The following problem is, thus, a weaker version of Problem 7.

Problem 8 Determine conditions under which the Petrie path of a chiral polytope is a polygon.

We shall refer to the Petrie paths of a chiral polytope $\mathcal{K}$ associated to the Petrie map $r_{d-1} r_{d-2} \cdots r_{0}$ by left Petrie paths, and those associated to the Petrie map $r_{d-1} r_{d-2} \cdots r_{2}$ $r_{0} r_{1}$ by right Petrie paths. Note that the length (number of edges) of the right Petrie paths of $\mathcal{K}$ correspond to the length of the left Petrie paths of its enantiomorphic form, and vice versa. Clearly, either all left (right) Petrie paths are polygons, or none of them is.

Problem 9 Is there a chiral polytope whose left Petrie paths are polygons, but whose right Petrie paths are not?

It was pointed out in [60, Section 3] that the lengths of the left and right Petrie paths of chiral polyhedra have the same length. This is true for $d$-polytopes whenever $d$ is not a multiple of 4 .

Proposition 4.2. The right and left Petrie paths of any chiral $d$-polytope have the same length for $d$ odd.

Proof. We claim that the group elements defining two steps on the right and left Petrie paths are conjugates. In fact, if $d=3$ this is straightforward; on the other hand, if $d=2 k+1$ then by Lemma 2.4 it follows that

$$
\begin{aligned}
& \sigma_{1}^{-1} \sigma_{3} \cdots \sigma_{2 k-1} \sigma_{1} \sigma_{2} \cdots \sigma_{2 k} \sigma_{1}^{2} \sigma_{2} \sigma_{4} \cdots \sigma_{2 k} \\
= & \sigma_{2} \sigma_{4} \cdots \sigma_{2 k-2}\left(\sigma_{1} \sigma_{2} \cdots \sigma_{2 k-1}\right)^{2} \sigma_{2 k} \sigma_{1} \sigma_{3} \cdots \sigma_{2 k-1} \sigma_{1} \sigma_{2} \cdots \sigma_{2 k} \\
= & \sigma_{2} \sigma_{4} \cdots \sigma_{2 k} \sigma_{1} \sigma_{3} \cdots \sigma_{2 k-1} \sigma_{1} \sigma_{2} \cdots \sigma_{2 k} .
\end{aligned}
$$

Conjugating the last expression by $\left(\sigma_{2} \sigma_{4} \cdots \sigma_{2 k}\right)^{-1}$, we obtain the desired result.

Proposition 4.3. The right and left Petrie paths of any chiral $d$-polytope have the same length for $d \equiv 2$ modulo 4 .

Proof. We prove that the group element defining a step on a right Petrie path is conjugate to the inverse of the element defining a step on the left Petrie path.

First note that commutativity of $\sigma_{i}$ and $\sigma_{j}$ if $|i-j| \geq 3$ (see Lemma 2.1) implies that $\sigma_{1}^{-1} \sigma_{3} \sigma_{5} \cdots \sigma_{d-1}$ is a conjugate of

$$
\sigma_{d-1} \sigma_{d-3} \cdots \sigma_{3} \sigma_{1}^{-1} .
$$


We conjugate the element in (4.1) by $\sigma_{(d / 2)-1} \sigma_{(d / 2)-3} \cdots \sigma_{2}$ to obtain the element

$$
\sigma_{d-1} \sigma_{d-3} \cdots \sigma_{(d / 2)+2} \sigma_{d / 2}^{-1} \sigma_{(d / 2)-2} \cdots \sigma_{1}
$$

(see Lemma $2.2(2)$ ).

By conjugating the element in (4.2) by the element $\tau=\sigma_{1} \cdots \sigma_{d-1}$ (see Lemma 2.2 (1)) we obtain

$$
\sigma_{d-1}^{-1} \sigma_{d-3} \sigma_{d-5} \cdots \sigma_{(d / 2)+2} \sigma_{d / 2}^{-1} \sigma_{(d / 2)-2} \sigma_{(d / 2)-4} \cdots \sigma_{3} \sigma_{1}^{-1} .
$$

By a suitable modification of the previous two steps it follows that $\sigma_{1}^{-1} \sigma_{3} \sigma_{5} \cdots \sigma_{d-1}$ is a conjugate of the expression obtained by replacing each $\sigma_{i}$ and $\sigma_{d-i}$ by $\sigma_{i}^{-1}$ and $\sigma_{d-i}^{-1}$ respectively. As a consequence, this expression is a conjugate of $\sigma_{2 d-1}^{-1} \sigma_{2 d-3}^{-1} \cdots \sigma_{1}^{-1}$, which is the inverse of the relation defining the left Petrie path.

There are examples of chiral 4-polytopes with the property that the left and right Petrie paths have different lengths (see [17], [51] and [58]). No such example is known for $4 k$ polytopes with $k \geq 2$.

Problem 10 Find examples of chiral $4 k$-polytopes (where $k \geq 2$ ) with the property that the length of the right Petrie paths does not coincide with the length of the left Petrie paths, or prove their non-existence.

The examples of chiral polytope-like structures of rank 4 with right Petrie paths of length $m$ and left Petrie paths of length $n<m$ given in [17] yield the pairs

$$
(m, n) \in\{(3,2),(4,2),(5,3),(6,3),(5,4),(9,5),(18,6),(30,8)\} .
$$

However, the structures corresponding to the first 5 pairs are not polytopes. In [58] the authors prove the existence of infinite families of chiral polytopes using projective linear groups. The length of the right and left Petrie paths is the order of a matrix, which can be found by a recursive procedure. The infinite family of chiral 4-polytopes described in [51] contains polytopes with right Petrie paths of length 4 and left Petrie paths of length $4 k$ for each $k \geq 2$.

Problem 11 Determine the pairs $(m, n)$ for which there exists a chiral 4-polytope with right Petrie paths of length $m$ and left Petrie paths of length $n$.

It is also interesting to determine the existence and properties of chiral 4-polytopes with the property that the lengths of the right and left Petrie paths coincide.

Problem 12 Determine the integers $n$ for which there exists a chiral 4-polytope with right and left Petrie paths of length $n$.

\section{Topological considerations}

We say that a regular $d$-polytope $\mathcal{K}$ is spherical whenever it is isomorphic to a regular tessellation of the $(d-1)$-sphere, or equivalently, it is isomorphic to the face lattice of a convex regular polytope. There are spherical regular $d$-polytopes for all rank $d \geq 2$. We say that $\mathcal{K}$ is Euclidean (resp. hyperbolic) whenever it is isomorphic to the face lattice of a geometrically regular tessellation of an Euclidean (resp. hyperbolic) $(d-1)$-space. 
Euclidean regular $d$-polytopes exist for each $d \geq 2$, whereas hyperbolic regular $d$-polytopes exist only for $d=3,4,5$. By a tessellation we understand a locally finite decomposition of the space into compact tiles, each of them isomorphic to a convex polytope (see [37, Chapter 6]). In this paper the terms "Euclidean polytope" and "hyperbolic polytope" are used exclusively to describe tessellations of the full Euclidean or hyperbolic space, not quotients of the latter.

Following this idea, we can define a spherical chiral polytope as the face lattice of a convex chiral polytope, and an Euclidean (resp. hyperbolic) chiral polytope as a chiral tessellation of an Euclidean (resp. hyperbolic) space. However, there exist no chiral, Euclidean or hyperbolic chiral polytopes (see for example [33, Theorem 11.2]).

A space-form is a quotient of the (spherical, Euclidean or hyperbolic) $d$-dimensional space $E$ by a discrete group of isometries which acts freely on $E$. The projective $d$-space and the $d$-torus are examples of spherical and Euclidean space forms respectively.

A polytope is locally spherical whenever its facets and vertex-figures are spherical. In [37, Chapter 6B] a general theory on locally spherical regular polytopes is provided, and every such regular polytope is shown to be a tessellation of a spherical, Euclidean or hyperbolic space-form arising as a quotient (from a combinatorial, geometric and topological point of view) of a regular tessellation of the spherical, Euclidean or hyperbolic space by a normal sparse subgroup. Similar arguments can be applied to prove an analogous result for chiral polytopes (see also [60, Section 5]).

In [37, Chapter 6C] it is shown that besides the spherical regular polytopes, the only regular tessellations of spherical space-forms are the projective regular $(n+1)$-polytopes in the projective $n$-space $\mathbb{P}^{n}$. The author does not know whether the existence or nonexistence of chiral tessellations of spherical space-forms has been established.

Problem 13 Determine whether there exist chiral tessellations of spherical space-forms.

For each $n \geq 2$ there exist infinite families of regular tessellations of the $n$-torus (see [37, Sections 6D, 6E]). Chiral tessellations of the 2-torus are classified in [19]. Later it was proved in [25, Section 4] that no other Euclidean space-form admits chiral tessellations (see also [37, Section $6 \mathrm{H}]$ ). In particular, all toroidal chiral $n$-polytopes (tessellation of the $n$-torus) have rank 3 .

The only locally finite tessellations of the hyperbolic $n$-space with convex regular polytopes are the universal polytopes with Schläfli type $\{p, q\}$, with $1 / p+1 / q<1 / 2$ for $n=2$; $\{4,3,5\},\{5,3,4\},\{5,3,5\}$ and $\{3,5,3\}$ for $n=3$; or $\{3,3,3,5\},\{5,3,3,3\},\{4,3,3,5\}$, $\{5,3,3,4\}$ and $\{5,3,3,5\}$ for $n=4$.

Little is known about regular or chiral tessellations on hyperbolic space-forms. Chiral tessellations of quotients of the hyperbolic plane are discussed in Section 6. The first examples of chiral tessellations of quotients of the hyperbolic 3 -space are described in [17, Table 3], and later also in [64]. Recently some examples of chiral tessellations of quotients of the hyperbolic 4- and 5-spaces were found [12].

Problem 14 Examine locally spherical chiral 5-polytopes of hyperbolic type.

Among all polytopes which are not locally spherical, special attention has been given to locally toroidal polytopes.

A polytope is locally toroidal whenever its facets and vertex-figures are spherical or toroidal, but not both spherical. 
Locally toroidal regular polytopes exist only for ranks $d=4,5,6$ (see [37, Chapter 10].

Whenever there exist chiral polytopes with facets isomorphic to a regular or chiral $(d-1)$-polytope $\mathcal{K}_{1}$ and vertex-figures isomorphic to a regular or chiral polytope $\mathcal{K}_{2}$, but not both $\mathcal{K}_{1}$ and $\mathcal{K}_{2}$ regular, there exists a universal polytope $\left\{\mathcal{K}_{1}, \mathcal{K}_{2}\right\}^{\text {ch }}$ with the property that every polytope with facets isomorphic to $\mathcal{K}_{1}$ and vertex-figures isomorphic to $\mathcal{K}_{2}$ is a quotient of $\left\{\mathcal{K}_{1}, \mathcal{K}_{2}\right\}^{\text {ch }}$ (see [60, Theorem 2]). This will be discussed in more detail in Section 7. Abusing notation, we may assume that $\Gamma^{+}\left(\mathcal{K}_{1}\right)=\left\langle\sigma_{1}, \ldots, \sigma_{d-2}\right\rangle$ subject to a certain set of relations $R_{1}$ and $\Gamma^{+}\left(\mathcal{K}_{2}\right)=\left\langle\sigma_{2}, \ldots, \sigma_{d-1}\right\rangle$ subject to a set of relations $R_{2}$. Then $\Gamma\left(\left\{\mathcal{K}_{1}, \mathcal{K}_{2}\right\}^{\text {ch }}\right)=\left\langle\sigma_{1}, \ldots, \sigma_{d-1}\right\rangle$ subject to the set of relations $R_{1} \cup R_{2}$ together with $\left(\sigma_{1} \sigma_{2} \cdots \sigma_{d-1}\right)^{2}=\varepsilon$ (see also [54] and [37, Section 4A]). In particular, this holds whenever $\mathcal{K}_{1}$ and $\mathcal{K}_{2}$ are spherical or toroidal.

The central two problems on locally toroidal polytopes are to determine whether $\left\{\mathcal{K}_{1}\right.$, $\left.\mathcal{K}_{2}\right\}^{\text {ch }}$ or $\left\{\mathcal{K}_{1}, \mathcal{K}_{2}\right\}$ (the regular equivalent of $\left\{\mathcal{K}_{1}, \mathcal{K}_{2}\right\}^{\text {ch }}$ ) exists, and if it does, whether it is finite. In the regular case, the classification of all spherical or toroidal $\mathcal{K}_{1}$ and $\mathcal{K}_{2}$ for which there exist polytopes with facets of type $\mathcal{K}_{1}$ and vertex-figures of type $\mathcal{K}_{2}$ is almost complete. In case of existence, in most of the cases it is known whether $\left\{\mathcal{K}_{1}, \mathcal{K}_{2}\right\}$ is finite or infinite, however certain cases remain open (see [37, Chapters 10, 11, 12], [42], [43], [44], [45]).

The same problem can be stated for locally toroidal chiral polytopes.

Since there are no spherical chiral polytopes and there are toroidal chiral polytopes only of rank 3, then any locally toroidal polytope with chiral facets and/or vertex-figures must have rank 4 . Some progress in this case can be found in [8].

The following two problems were stated as Problem 19 in [60].

Problem 15 Determine all spherical or toroidal $(d-1)$-polytopes $\mathcal{K}_{1}$ and $\mathcal{K}_{2}$ such that at least one of $\mathcal{K}_{1}$ or $\mathcal{K}_{2}$ is chiral and there exists a chiral $d$-polytope with facets isomorphic to $\mathcal{K}_{1}$ and vertex-figures isomorphic to $\mathcal{K}_{2}$.

Problem 16 Determine all finite universal locally toroidal polytopes.

We can still consider the case where both spherical or toroidal $(d-1)$-polytopes are regular. In this case, whenever it exists, the corresponding universal locally toroidal polytope must be regular.

Problem 17 Determine all spherical $(d-1)$-polytopes $\mathcal{K}_{1}$ and $\mathcal{K}_{2}$ for which there exists a chiral $d$-polytope with facets isomorphic to $\mathcal{K}_{1}$ and vertex-figures isomorphic to $\mathcal{K}_{2}$.

\section{$6 \quad$ Rank 3}

All polyhedra (rank 3 polytopes) are locally spherical (see Section 5) since both the facets and vertex-figures are isomorphic to convex polygons. It follows that each finite polyhedron can be represented as a tessellation on a compact surface without boundary.

A map is a 2-cell embedding of a graph on a surface. Maps have been broadly studied (see for example [39]). Note that not every map satisfies the diamond condition, and therefore not all maps are 3-polytopes.

The definitions of flags, automorphisms, regularity and chirality extend naturally from polytopes to maps. Regular and chiral maps have been extensively studied since the early 20th century ([3]). All chiral maps on the torus were described by Coxeter in [19, Section 3.2]. They are classified in three families, each of which contains maps with the same 
Schläfli type $\{3,6\},\{4,4\}$ or $\{6,3\}$. Several years later chiral maps on surfaces with higher genus were discovered. Edmonds found a map with type $\{7,7\}$ on an orientable surface with genus 7. This map is described in [16, Chapter 21.3]. Sherk constructed a family of chiral maps with type $\{6,6\}$ ([61]), and Garve proved that there is no chiral map on surfaces with genus $2,3,4,5$ or 6 ([21]). Since then, several chiral maps on surfaces with higher genus have been found (see [66], [13], [46] and [11]).

Regular and chiral maps have been considered from the viewpoints of the surfaces (see for example [6], [11] and [13]), of the automorphism groups (see [46]) and of the graphs (see [7], [28] and[62]). However little has been said about which regular and chiral maps are polyhedra. In each orientable surface with genus 2 or greater there exists a regular map with one vertex. Clearly, this is not a polyhedron. Similarly, several chiral maps are not polyhedra, such as the map C11.4 in [11].

It would be convenient to know whether a result for polytopes extends for maps, and also which results for maps translate directly to polytopes. This motivates the following problem.

Problem 18 Characterize chiral maps which are not polyhedra.

Residual finiteness was used in [63, Theorem 6.3] to prove that given any Schläfli type $\{p, q\}$ with $\frac{1}{p}+\frac{1}{q} \leq \frac{1}{2}$, there are infinitely many regular maps whose faces are $p$ gons, $q$ of them around each vertex. Similar techniques can be used to prove that there are infinitely many finite chiral maps (and even chiral polytopes) with Schläfli type $\{p, q\}$ with $\frac{1}{p}+\frac{1}{q} \leq \frac{1}{2}$. However, given a surface $S$ with negative Euler characteristic there exist at most finitely many regular and chiral maps on $S$ (see for example [13]).

No chiral map lies on a non-orientable surface (see for example [13]). Also, as noted above, there are no chiral maps on orientable surfaces from genus 2 to genus 6 . Furthermore, it was proved in [15, Lemma 6.2] that orientable surfaces with genera $p+1$, with $p$ prime and $p-1$ not divisible by 3,5 or 8 , do not admit chiral maps. This result implies that there are infinitely many genera $g$ such that there are no chiral maps on orientable surfaces with genus $g$.

Problem 19 Determine all positive integers $g$ for which there are chiral maps (polyhedra) on orientable surfaces with genus $g$.

In orientable surfaces with small genera, regular maps appear to be more numerous than chiral maps. For instance, on surfaces of genera $2 \leq g \leq 5$ there are 220 regular maps and only 16 chiral maps (see [13]). However, as we increase the genus, apparently the number of chiral polytopes grows faster than the number of regular polytopes. For instance, on orientable surfaces of genera $2 \leq g \leq 101$ there are 3378 regular maps and 594 chiral maps (see [11]).

Problem 20 Let $n_{\mathrm{r}}(g)$ and $n_{\mathrm{ch}}(g)$ denote respectively the number of regular and chiral polytopes on orientable surfaces of genus at most $g$. Determine

$$
\lim _{g \rightarrow \infty} \frac{n_{\mathrm{r}}(g)}{n_{\mathrm{ch}}(g)}
$$

if the limit exists.

Some infinite regular polyhedra arise naturally as tessellations of the Euclidean or hyperbolic plane. In fact, we can construct one of them for each Schläfli type $\{p, q\}$ with $\frac{1}{p}+\frac{1}{p} \leq \frac{1}{2}$. 
Very little is known about infinite chiral polyhedra. Permutation groups were used in [51] to find chiral polyhedra with Schläfli symbols $\{8,4\},\{\infty, 6\}$ and $\{\infty, \infty\}$. Chiral polyhedra with Schläfli types $\{6,6\},\{6,4\}$ and $\{4,6\}$ were found in [55] as geometric objects in $\mathbb{E}^{3}$, and proved to be combinatorially chiral in [50] (see Section 7).

Problem 21 Determine all pairs $\{p, q\}$ with $p, q \in\{3,4, \ldots\} \cup\{\infty\}$ for which there exist infinite chiral maps (polyhedra) with Schläfli type $\{p, q\}$.

The classification of noncompact surfaces was achieved in [52]. Whenever an infinite polyhedron has finite faces and vertex-figures, it can be understood like a map on a noncompact surface where the faces are considered to be discs, two of them identified in a line segment in the boundary whenever they share an edge. The identifications must be made in such a way that the cyclic order of the identifications around a face and around a vertex are the same as the order of the corresponding edges in the polyhedron. The next problem follows the spirit of Problem 19.

Problem 22 Determine which noncompact surfaces without boundary admit embeddings of chiral maps.

\section{Higher ranks}

Convex regular polytopes, regular tessellations of the Euclidean spaces and the regular toroidal quotients of the latter are natural examples of regular polytopes of each rank $d \geq 3$. In contrast, only sporadic examples of chiral $d$-polytopes are known to exist for $d \geq 4$. That is, there is no "natural" construction known of chiral polytopes of each rank. Moreover, for some time it was not even clear that there exist chiral polytopes of each rank $d \geq 3$.

The first examples of chiral structures in the sense of abstract polytopes of rank greater than 3 are due to Coxeter, and were obtained as quotients of regular tessellations of the hyperbolic 3-space, by forcing the right and left Petrie walks to have distinct lengths (see [17] and Sections 4, 5). More examples of this type were found in [47], [64] and [58]. In the latter, instead of tessellations with compact tiles, finitely many of which meet at every vertex, the honeycombs $\{4,4,3\},\{4,4,4\},\{6,3,3\},\{3,6,3\}$ and $\{6,3,6\}$ are considered. In each case, the faces are infinite (non-convex) polyhedra, whose vertices lie on a horosphere. The vertex-figures may be either convex regular polyhedra, or again infinite polyhedra with vertices on a horosphere.

Besides the locally finite tessellations of the hyperbolic $n$-space with convex regular tiles (see Section 5) and the five honeycombs mentioned in the previous paragraph together with their duals, we may consider the honeycombs $\{6,3,4\},\{6,3,5\}$ and their duals in hyperbolic 3 -space, the honeycombs $\{3,4,3,4\}$ and $\{4,3,4,3\}$ in hyperbolic 4 -space, and the honeycombs $\{3,3,4,3,3\},\{3,3,3,4,3\},\{3,4,3,3,3\},\{4,3,3,4,3\}$ and $\{3,4,3,3,4\}$ in hyperbolic 5 -space. Recently some examples of chiral quotients of these honeycombs were found [10].

Problem 23 Determine the existence of infinite families of chiral quotients of all regular honeycombs in hyperbolic 3-, 4- and 5-space.

One procedure of constructing polytopes of rank $d$ is to determine whether it is possible to assemble a $d$-polytope from a set of $(d-1)$-polytopes as facets of a prescribed type. Whenever the facets are all isomorphic, the $d$-polytope is known as an extension of the $(d-1)$-polytope. 
In [53] it was proved that for every regular $d$-polytope $\mathcal{K}$ there exists a universal regular extension $U(\mathcal{K})$ with the following properties.

(a) Every other regular extension of $\mathcal{K}$ is a quotient of the $U(\mathcal{K})$;

(b) $U(\mathcal{K})$ is an infinite polytope, and the last entry of its Schläfli type is $\infty$;

(c) the automorphism group of $U(\mathcal{K})$ is obtained from $\Gamma(\mathcal{K})$ by adding an extra involutory generator $\rho_{d}$ satisfying relations (2.2).

The equivalent result for chiral polytopes was established in [59]. Specifically, it was established that every chiral $d$-polytope $\mathcal{K}$ with regular facets admits a universal chiral extension $U^{c h}(\mathcal{K})$ satisfying the equivalent to (a) and (b) as for the universal regular extension of a regular polytope, with the property that the automorphism group of $U^{c h}(\mathcal{K})$ is obtained from $\Gamma(\mathcal{K})$ by adding an extra generator $\sigma_{d}$ with the extra relations given by (2.4).

If $\mathcal{K}$ is orientably regular, the polytope obtained from $\Gamma^{+}(\mathcal{K})$ by adding a generator $\sigma_{d}$ and relations (2.4) is its universal regular cover $U(\mathcal{K})$. In this sense, $U^{c h}(\mathcal{K})$ does not exist for regular $\mathcal{K}$. However, it is not known yet whether there exists a chiral polytope covering all other chiral extensions of a regular polytope $\mathcal{K}$.

Problem 24 Determine whether for every (any) regular polytope $\mathcal{K}$ there exists a chiral extension which covers every extension of $\mathcal{K}$.

The requirement of regularity for the facets of $\mathcal{K}$ is necessary for the existence of $U^{c h}(\mathcal{K})$ since the facets of the extension are isomorphic to $\mathcal{K}$. Therefore, this construction cannot be applied twice. However, if $\mathcal{K}$ is a chiral $d$-polytope with regular facets and vertex-figures, we may obtain the infinite chiral $(d+2)$-polytope $\left(U\left(U(\mathcal{K})^{*}\right)\right)^{*}$, the dual of the universal extension of the dual of the universal extension of $\mathcal{K}$. This result can be applied to chiral 4-polytopes with regular facets and vertex-figures (for example, those in [47]) to obtain infinite chiral 5- and 6-polytopes.

The first finite chiral 5-polytopes were found in [14] with the aid of a computer. Later similar methods were used to find finite chiral $d$-polytopes for $d=6,7,8$ [12]. (A computer based method was also used to construct the atlas in [24].) Then a proof of existence of finite $d$-polytopes for every rank $d \geq 3$ was given in [49], providing a positive answer to Problem 14 in [60].

There are regular extensions of each regular polytope $\mathcal{K}$. Furthermore, if $\mathcal{K}$ is finite, it admits finite regular extensions (see for example [20] and [53]). The Schläfli type of any equivelar extension of an equivelar polytope $\mathcal{K}$ will coincide on the first $d-1$ entries with the Schläfli type of $\mathcal{K}$. It was determined in [48] that every regular polytope admits regular extensions where the last entry of the Schlälfi type is an arbitrary even number $p_{d}$. On the other hand, Hartley proved in [23] that the hemicube does not admit regular extensions with odd numbers as last entries on their Schläfli symbols.

Little is known about chiral extensions of regular or chiral polytopes. The main result in this direction is the construction of $U^{c h}(\mathcal{K})$ given in [59]. It is natural then to ask the following questions.

Problem 25 Does every chiral polytope $\mathcal{K}$ with regular facets admit a chiral extension with prescribed last entry of the Schläfli symbol?

Problem 26 Does every chiral finite polytope $\mathcal{K}$ with regular facets admit a finite chiral extension with prescribed last entry of the Schläfli symbol? 
A necessary condition for a regular polytope to admit chiral extensions is orientability. The only known general result in this direction is given in [49], where chiral extensions were found for minimal regular covers of the so-called scattered chiral polytopes. This was used to provide a recursive construction of chiral polytopes of all ranks higher than 3 . However, very few polytopes are regular covers of scattered chiral polytopes.

Problem 27 Does every orientably regular $d$-polytope admit a chiral extension $(d \geq 2)$ ?

Problem 28 Does every orientably regular finite $d$-polytope admit a finite chiral extension $(d \geq 2)$ ?

We recall that every chiral polytope $\mathcal{K}$ with regular facets admits an infinite chiral extension, namely $U^{c h}(\mathcal{K})$. It is interesting to determine whether this is true for orientably regular polytopes.

Problem 29 Does every orientably regular polytope admit an infinite chiral extension?

Problems 27, 28 and 29 seem hard to answer, and some partial results would be of interest. Chiral polytopes with a $d$-simplex as facet were found by Conder and Devillers for $d=6,7,8$ [12]. The next problem is a general version of the dual of Problem 13 of [60].

Problem 30 Does every convex regular polytope admit a chiral extensions?

Given two $(d-1)$-polytopes $\mathcal{K}_{1}$ and $\mathcal{K}_{2}$, we say that a $d$-polytope $\mathcal{P}$ is an amalgamation of $\mathcal{K}_{1}$ and $\mathcal{K}_{2}$ whenever all of its facets are isomorphic to $\mathcal{K}_{1}$ and all of its vertex-figures are isomorphic to $\mathcal{K}_{2}$ (see [37, Chapter 4]). In other words, an amalgamation of $\mathcal{K}_{1}$ and $\mathcal{K}_{2}$ is an extension of $\mathcal{K}_{1}$ where we are additionally prescribing the type of vertex-figure to be $\mathcal{K}_{2}$.

In general it is hard to determine whether there exist amalgamations of two given polytopes $\mathcal{K}_{1}$ and $\mathcal{K}_{2}$. A necessary condition is that the vertex-figures of $\mathcal{K}_{1}$ are isomorphic to the facets of $\mathcal{K}_{2}$, but this condition is not sufficient. Whenever there exists a chiral amalgamation of two polytopes $\mathcal{K}_{1}$ and $\mathcal{K}_{2}$ such that at least one $\mathcal{K}_{1}$ and $\mathcal{K}_{2}$ is chiral and the other one is regular or chiral, there exists a universal amalgamation $\left\{\mathcal{K}_{1}, \mathcal{K}_{2}\right\}^{\text {ch }}$ with the property that any other amalgamation of $\mathcal{K}_{1}$ and $\mathcal{K}_{2}$ is a quotient of it (see [57, Section 6]). We include here the chiral part of Problem 8 and Problem 11 of [60].

Problem 31 Given regular (or chiral) $(d-1)$-polytopes $\mathcal{K}_{1}$ and $\mathcal{K}_{2}$, does there exist a chiral $d$-polytope $\mathcal{P}$ with faces isomorphic to $\mathcal{K}_{1}$ and vertex-figures isomorphic to $\mathcal{K}_{2}$ ?

Problem 32 Determine the group of the universal amalgamation $\left\{\mathcal{K}_{1}, \mathcal{K}_{2}\right\}^{\text {ch }}$ and give conditions under which this is finite, where $\mathcal{K}_{1}$ and $\mathcal{K}_{2}$ are regular or enantiomorphic forms of chiral polytopes, not both regular.

A polytope $\mathcal{K}$ is said to be (combinatorially) flat whenever every facet contains every vertex. The hemi-cube and hemi-octahedron are examples of flat polytopes. More generally, a polytope is said to be $(k, m)$-flat whenever every $m$-face contains every $k$ face (see [37, Section $4 \mathrm{E}])$. A $(k, m)$-flat polytope is also a $(l, n)$-flat polytope for every $0 \leq l \leq k<m \leq n \leq d-1$.

Chiral flat 3-, 4- and 5-polytopes are known to exist. The polyhedron C7.1 in [11] 
with Schläfli type $\{6,9\}$ is flat. In [14, Section 4] a dual pair or chiral flat 4-polytopes with Schläfli types $\{3,4,4\}$ and $\{4,4,3\}$ are described. Furthermore, in [51, Section 6.1] a chiral flat 4-polytope with Schläfli type $\{4,3,6 q\}$ is constructed for each $q \geq 2$. An example of a chiral $(0,2)$-flat 5-polytope is given in [14, Section 5]. Its Schläfli type is $\{3,6,3,6\}$.

Problem 33 Does there exist a flat $d$-polytopes for every $d \geq 3$ ?

Problem 34 Does there exist a $(k, m)$-flat $d$-polytopes for each triple $(k, m, d)$ with $0<k+1<m \leq d-1$ ?

To conclude this section we discuss regular and chiral $d$-polytopes with the fewest possible flags.

Let $m_{d}^{\mathrm{r}}$ and $m_{d}^{\text {ch }}$ denote the minimum of the numbers of flags of all regular and chiral $d$-polytopes respectively. Then clearly $m_{d}^{\mathrm{r}} \leq(n+1)$ ! since the $d$-simplex has precisely this number of flags. Recently $m_{d}^{\mathrm{r}}$ was established for all $d$, with the value of $\left(4^{d}\right) / 2$ for $d \geq 9$ [9]. The number $m_{d}^{\text {ch }}$ seems to grow much faster than $m_{d}^{\mathrm{r}}$, however little is known about it. The construction of chiral polytopes of ranks $d \geq 4$ in [49] is recursive, and therefore it does not provide the function itself. An upper bound provided by this construction is given by the recursive function $f_{c h}$ where $f_{c h}(3)=84$ and $f_{c h}(n+1)=$ $\left[\left(2 f_{c h}(n)+1\right)\left(f_{c h}(n) !\right)^{2}\right]$ !. However this bound may be very far away from the value of $m_{d}^{\mathrm{ch}}$.

Problem 35 Find the value of $m_{d}^{\text {ch }}$ as a function of $d$.

\section{Realizations}

Following [37, Section 5A], a realization of an abstract polytope $\mathcal{K}$ in a geometric space $S$ is a mapping $\beta=\beta_{0}$ from the vertex set of $\mathcal{K}$ into $S$. This indicates which points of $S$ correspond to the vertices of the realization of $\mathcal{K}$. The image of the remaining faces of $\mathcal{K}$ are defined recursively in the following way. For $i=1, \ldots, d-1$ define $\beta_{i}$ from the sets of $i$-faces of $\mathcal{K}$ to the power set of the set of images in $S$ of the $(i-1)$-faces of $\mathcal{K}$, such that for each $i$-face $F$ of $\mathcal{K}$,

$$
\beta_{i} F=\left\{\beta_{i-1} G \mid G \subseteq F\right\} .
$$

We say that a realization is faithful if $\beta_{i}$ is one-to-one for all $i$. The image of $\mathcal{K}$ under a realization is called a geometric polytope.

A symmetry of the geometric polytope $\mathcal{P}$ is an isometry of $S$ that preserves $\mathcal{P}$. A realization $\mathcal{P}$ of a polytope $\mathcal{K}$ is said to be symmetric if each automorphism of $\mathcal{K}$ induces a symmetry of $\mathcal{P}$. Note that if $\mathcal{P}$ is not symmetric, then the symmetry group of $\mathcal{P}$ is isomorphic to a proper subgroup of $\Gamma(\mathcal{K})$.

A geometric polytope $\mathcal{P}$ is said to be geometrically regular if its symmetry group is transitive on its flags. Whenever $\mathcal{P}$ is a faithful realization of the abstract polytope $\mathcal{K}$, the symmetry group of $\mathcal{P}$ is isomorphic to the automorphism group of $\mathcal{K}$, implying that $\mathcal{K}$ is also regular as an abstract polytope.

A geometric polytope $\mathcal{P}$ is said to be geometrically chiral if its symmetry group has two orbits on its flags in such a way that adjacent flags are in distinct orbits. The symmetry group of a faithful geometrically chiral realization $\mathcal{P}$ of an abstract polytope $\mathcal{K}$ is isomorphic to a subgroup of the automorphism group of $\mathcal{K}$ of index at most 2 . If the index is 2 then 
$\mathcal{K}$ is regular as an abstract polytope, and the automorphisms represented by the symmetries are precisely the elements of $\Gamma^{+}(\mathcal{K})$. We also say that $\mathcal{K}$ admits a chiral realization. On the other hand, if the index is 1 then $\mathcal{K}$ is chiral as an abstract polytope. Consequently, if a polytope admits a chiral realization it must be either chiral or orientably regular.

The study of realizations of polytopes involves two main branches: the description of all realizations of a given polytope, and the determination of all polytopes realizable on a given space.

For regular realizations, the first branch was thoroughly studied and is described in [37, Chapter 5]. In the finite case, the set of all regular realizations of a given regular polytope $\mathcal{P}$ has the structure of a convex cone. The structure of the cone is determined by the family of irreducible orthogonal representations of the automorphism group of $\mathcal{P}$. No such theory has been developed for chiral realizations of regular or chiral polytopes. We include here Problem 21 of [60].

Problem 36 Develop the details of the realization theory for chiral polytopes.

In [36] McMullen and Schulte provide a survey of all the geometrically regular discrete polyhedra in $\mathbb{E}^{3}$ (see also [37, Section 7E]). There are 18 finite regular polyhedra, 6 infinite regular polyhedra in the plane, and 24 infinite geometrically regular polyhedra such that the affine hull of their vertices is $\mathbb{E}^{3}$. The latter are divided in two families: 12 blended polyhedra having reducible symmetry groups (the groups permutes a linear subspace of $\mathbb{E}^{3}$ and its translates), and 12 pure polyhedra having irreducible symmetry groups. Furthermore, they also classify the 8 regular 4 -polytopes in $\mathbb{E}^{3}$.

A geometric realization $\mathcal{P}$ of an abstract $d$-polytope $\mathcal{K}$ is said to be of full rank whenever either $\mathcal{P}$ is finite and its ambient space is the Euclidean $d$-space $\mathbb{E}^{d}$, or $\mathcal{P}$ is infinite and its ambient space is $\mathbb{E}^{d-1}$. Full rank regular polytopes were classified in [33].

Discrete (finite) regular polytopes in the projective $d$-space $\mathbb{P}^{d}$ are in correspondence with finite polytopes in the Eudlidean $(d+1)$-space. All discrete polyhedra with planar faces in the projective space $\mathbb{P}^{3}$ were found in [1] and [2]. The enumeration was completed in [34], where the author determined all regular finite polyhedra in the Euclidean 4-space $\mathbb{E}^{4}$. The classification of discrete regular 4-apeirotopes in $\mathbb{E}^{4}$ was achieved in [35].

Much less has been achieved for geometrically chiral polytopes.

It was proved in [33] that there are no geometric chiral polytopes of full rank. In particular, there are no finite chiral polyhedra in $\mathbb{E}^{3}$.

The complete classification of discrete chiral geometric polyhedra in $\mathbb{E}^{3}$ was achieved in [55] and [56]. Every chiral polyhedron in $\mathbb{E}^{3}$ belongs to one of the 6 infinite families $\{P(a, b)\},\{Q(c, d)\},\left\{Q(c, d)^{*}\right\},\left\{P_{1}(a, b)\right\},\left\{P_{2}(c, d)\right\}$ and $\left\{P_{3}(c, d)\right\}$, each indexed by two parameters.

The families $P(a, b), Q(c, d)$ and $Q(c, d)^{*}$ contain polyhedra with finite faces and Schläfli types $\{6,6\},\{4,6\}$ and $\{6,4\}$ respectively. Any of the parameters $a, b, c$ and $d$ may be 0 ; otherwise $a$ and $c$ are rational multiples of the real parameters $b$ and $d$ respectively. Furthermore, $P(a, b), Q(c, d)$ and $Q(c, d)^{*}$ are respectively similar to $P(s a, s b)$, $Q(s c, s d)$ and $Q(s c, s d)^{*}$ for every real $s \neq 0$. Therefore the parameters $a, b, c$ and $d$ can be thought as integers. For pairs $(a, b)$ and $\left(a^{\prime}, b^{\prime}\right)$ of relatively prime integers with $a \geq 0$ and $(a, b) \neq \pm\left(a^{\prime}, b^{\prime}\right)$, the polyhedron $P(a, b)$ is not isomorphic (as an abstract polytope) to $P\left(a^{\prime}, b^{\prime}\right)$. Similarly, for $(c, d) \neq \pm\left(c^{\prime}, d^{\prime}\right)$ the polyhedra $Q(c, d)$ and $Q(c, d)^{*}$ are not isomorphic to $Q\left(c^{\prime}, d^{\prime}\right)$ and $Q\left(c^{\prime}, d^{\prime}\right)^{*}$ respectively.

The families $P_{1}(a, b), P_{2}(c, d)$ and $P_{3}(c, d)$ contain polyhedra with infinite helical faces 
and Schläfli type $\{\infty, 3\},\{\infty, 3\}$ and $\{\infty, 4\}$ respectively. The parameters $a, b, c$ and $d$ are any real numbers, and hence the set of parameters is not discrete (like it is for the families containing polyhedra with finite faces).

In [50] it was proved that all chiral polyhedra $P(a, b), Q(c, d)$ and $Q(c, d)^{*}$ are chiral as abstract polytopes. This provides infinitely many examples of infinite chiral polyhedra with the same Schläfli type.

On the other hand, all chiral polyhedra $P_{i}(a, b), i=1,2,3$ are regular as abstract polytopes. Furthermore, all geometrically chiral instances of $P_{1}(a, b), P_{2}(c, d)$ and $P_{3}(c, d)$ are respectively isomorphic to the regular polyhedra $\{\infty, 3\}^{(a)},\{\infty, 3\}^{(b)}$ and the orientable double cover of $\{\infty, 4\} ., * 3$ (in the notation of [37, Section 7E]), where a continuous change on the parameters corresponds to a continuous movement of the vertices and edges of the polyhedron. This provided the first examples of faithful discrete chiral realizations of regular polytopes.

The only known classifications of chiral polytopes in a given Euclidean space arise from the results just mentioned. This suggests the following problem.

Problem 37 Classify all finite and infinite chiral $k$-polytopes in $R^{d}$ (where $k<d$ for finite polytopes, and $k \leq d$ for infinite ones).

Of special interest is the case $d=4$ for both, finite rank 3 polytopes and infinite rank 4-polytopes. These two instances are not known for chiral, but have been settled for the regular case.

Problem 38 Classify all finite chiral polyhedra in $\mathbb{E}^{4}$.

Problem 39 Classify all infinite chiral 4-polytopes in $\mathbb{E}^{4}$.

\section{Acknowledgements}

The author is very grateful to Marston Conder, Egon Schulte, and Asia Weiss for some helpful ideas and discussion. He also wishes to thank the anonymous referees for a number of helpful suggestions.

\section{References}

[1] J. L. Arocha, J. Bracho and L. Montejano, Regular projective polyhedra with planar faces, I, Aequationes Math. 59 (2000), 55-73.

[2] J. Bracho, Regular projective polyhedra with planar faces, II, Aequationes Math. 59 (2000), 160-176.

[3] H. R. Brahana, Regular maps and their groups, Amer. J. Math. 49 (1927), 268-284.

[4] A. Breda, G. A. Jones, R. Nedela and M. Škoviera, Chirality groups of maps and hypermaps, J. Algebraic combinatorics 29 (2009), 337-355.

[5] A. Breda, G. A. Jones and E. Schulte, Constructions of chiral polytopes of small rank, manuscript.

[6] E. Bujalance, M. Conder and A. Costa, Pseudo-real Riemann surfaces and chiral regular maps, Trans. Amer. Math. Soc. 7 (2010), 3365-3376.

[7] D. Catalano and R. Nedela, A characterization of regular embeddings of $n$-dimensional cubes, Discrete Math. 310 (2010), 2364-2371. 
[8] C. J. Colbourn and A. I. Weiss, A census of regular 3-polystroma arising from honeycombs, Discrete Math.50 (1984), 29-36.

[9] M. Conder, Regular polytopes with the fewest flags, (in preparation).

[10] M. Conder, (private communication, 2010).

[11] M. Conder, Regular maps and hypermaps of Euler characteristic -1 to -200 , J. Combin. Theory Ser. B 99 (2009), 455-459, with associated lists available at http: / / www . math . auckland.ac.nz/ conder/.

[12] M. Conder and A. Devillers, (private communication, 2009).

[13] M. Conder and P. Dobcsányi, Determination of all regular maps of small genus, J. Combin. Theory Ser. B 81 (2001), 224-242.

[14] M. Conder, I. Hubard and T. Pisanski, Constructions for chiral polytopes, J. London Math. Soc. (2) 77 (2008), 115-129.

[15] M. Conder, J. Širáň and T. Tucker, The genera, reflexibility and simplicity of regular maps, J. Eur. Math. Soc. (JEMS) 12 (2010), 343-364.

[16] H. S. M. Coxeter, Introduction to geometry (2nd Ed.), Wiley, New York, 1969.

[17] H. S. M. Coxeter, Twisted honeycombs, CBMS Regional Conference Series in Mathematics No. 4, American Mathematical Society, Providence, RI, 1970.

[18] H. S. M. Coxeter, Regular Polytopes (3rd edition), Dover, New York, 1973.

[19] H. S. M. Coxeter and W. O. J. Moser, Generators and Relations for Discrete Groups, 4th edition, Springer-Verlag, Berlin-New York, 1980.

[20] L. Danzer, Regular incidence-complexes and dimensionally unbounded sequences of such, I, Convexity and Graph Theory (Jerusalem 1981), North-Holland Math. Stud. 87, North-Holland (Amsterdam, 1984), 115-127.

[21] D. Garbe, Uber die regulären Zerlegungen geschlossener orientierbarer Flächen, J. Reine Angew. Math. 237 (1969), 39-55.

[22] M. I. Hartley, All polytopes are quotients, and isomorphic polytopes are quotients by conjugate subgroups, Discrete Comput. Geom. 21 (1999), 289-298.

[23] M. I. Hartley, An exploration of locally projective polytopes, Combinatorica 28 (2008), no. 3, 299-314.

[24] M. Hartley, I. Hubard and D. Leemans, Two atlases of abstract chiral polytopes for small groups, manuscript.

[25] M. I. Hartley, P. McMullen and E. Schulte, Symmetric tessellations on euclidean space-forms, Canad. J. Math. (6) 51 (1999), 1230-1239.

[26] M. I. Hartley and G. Williams, Representing the sporadic Archimedean polyhedra as abstract polytopes, Discrete Math. 310 (2010), 1835-1844.

[27] I. Hubard, A. Orbanić and A. I. Weiss, Monodromy groups and self-invariance, Canad. J. of Math. 6 (2009), 1300-1324.

[28] G. Jones, Regular embeddings of complete bipartite graphs: classification and enumeration, Proc. Lond. Math. Soc. 101 (2010), 427-453.

[29] G. Jones and D. Singerman, Maps, hypermaps and triangle groups, The Grothendieck theory of dessins d'enfants, Luminy-1993, LMS Lecture Notes Series 200, Cambridge Univ. Press, Cambridge, 1994, 115-145.

[30] D. Leemans and E. Schulte, Groups of type $L_{2}(q)$ acting on polytopes, Adv. Geom. 7 (2007), 529-539. 
[31] D. Leemans and L. Vauthier, An atlas of abstract regular polytopes for small groups, http: //www.math.auckland.ac.nz/ dleemans/polytopes/, Aequationes Math. 72 (2006), 313-320.

[32] P. McMullen, Combinatorially regular polytopes, Mathematika 14 (1967), 142-150.

[33] P. McMullen, Regular polytopes of full rank, Discrete Comput. Geom. 32 (2004), 1-35.

[34] P. McMullen, Four-dimensional regular polyhedra, Discrete Comput. Geom. 38 (2007), 355387.

[35] P. McMullen, Regular apeirotopes of dimension and rank 4, Discrete Comput. Geom. 42 (2009), 224-260.

[36] P. McMullen and E. Schulte, Regular polytopes in ordinary space, Discrete Comput. Geom. 17 (1997), 449-478.

[37] P. McMullen and E. Schulte, Abstract regular polytopes, Encyclopedia of Math. And its Applic. 92, Cambridge, 2002.

[38] P. McMullen and E. Schulte, Regular and chiral polytopes in low dimensions, In The Coxeter Legacy - Reflections and Projections (eds. C. Davis and E. W. Ellers), Fields Institute Communications, Volume 48, American Mathematical Society, Providence, RI, 2006, 87-106.

[39] B. Mohar and C. Thomassen, Graphs on surfaces, Johns Hopkins University Press, Baltimore, MD, (2001).

[40] B. Monson, D. Pellicer and G. Williams, The tomotope, manuscript.

[41] B. Monson, D. Pellicer and G. Williams, Mixing and monodromy of abstract polytopes, in preparation.

[42] B. Monson and E. Schulte, Reflection groups and polytopes over finite fields, I, Adv. in Appl. Math. 33 (2004), 290-317.

[43] B. Monson and E. Schulte, Reflection groups and polytopes over finite fields, II, Adv. in Appl. Math. 38 (2007), 327-356.

[44] B. Monson and E. Schulte, Reflection groups and polytopes over finite fields, III, Adv. in Appl. Math. 41 (2008), 76-94.

[45] B. Monson and E. Schulte, Locally toroidal polytopes and modular linear groups, Discrete Math. 310 (2010), 1759-1771.

[46] R. Nedela and J. Širáň, Chiral maps of prescribed type arising from $A_{n}$, Abstracts of the Third Workshop in Symmetries in Graphs, Maps and Complexes (2006). Aveiro, Portugal.

[47] B. Nostrand, Ring extensions and chiral polytopes, Proceedings of the 25th Southeastern International Conference on Combinatorics, Graph Theory and Computing, Boca Raton, FL, 1994, Congr. Numer. 102 (1994), 147-153.

[48] D. Pellicer, Extensions of regular polytopes with preassigned Schläfli symbol, Journal of Combinatorial Theory, Series A 116 (2009), 303-313.

[49] D. Pellicer, A construction of higher rank chiral polytopes, Discrete Math. 310 (2010), 12221237.

[50] D. Pellicer and A. I. Weiss, Combinatorial Structure of Schulte's Chiral Polyhedra, Discrete Comput. Geom. 44 (2010), 167-194.

[51] D. Pellicer and A. I. Weiss, Generalized CPR graphs and applications, Contrib. Discrete Math. 5 (2010), 76-105.

[52] I. Richards, On the classification of noncompact surfaces, Trans. Amer. Math. Soc. 106 (1963), 259-269. 
[53] E. Schulte, On arranging regular incidence-complexes as faces of higher-dimensional ones, European Journal of Combinatorics 4 (1983), 375-384.

[54] E. Schulte, Amalgamation of regular incidence-polytopes, Proc. London Math. Soc. (3) 56 (1988), 303-328.

[55] E. Schulte, Chiral polyhedra in ordinary space, I, Discrete Comput. Geom. 32 (2004), 55-99.

[56] E. Schulte, Chiral polyhedra in ordinary space, II, Discrete Comput. Geom. 34 (2005), 181229.

[57] E. Schulte and A. I. Weiss, Chiral polytopes, Applied Geometry and Discrete Mathematics (The "Victor Klee Festschrift"), DIMACS Ser. Discrete Math. Theoret. Comput. Sci. 4 (1991), (eds. P. Gritzmann and B. Sturmfels), 493-516.

[58] E. Schulte and A. I. Weiss, Chirality and projective linear groups, Discrete Math. 131 (1994), 221-261.

[59] E. Schulte and A. I. Weiss, Free extensions of chiral polytopes, Canadian Journal of Math 47 (1995), 641-654.

[60] E. Schulte and A. I. Weiss, Problems on polytopes, their groups, and realizations, Periodica Matematica Hungarica 53 (2006), 231-255.

[61] F. A. Sherk, A family of regular maps of type $\{6,6\}$, Canadian Math. Bull. 5 (1962), 13-20.

[62] C. Thomassen, Tilings of the torus and the Klein bottle and vertex-transitive graphs on a fixed surface, Trans. Amer. Math. Soc. 323 (1991), 605-635.

[63] A. Vince, Regular combinatorial maps, J. Combin. Theory Ser. B 35 (1983), 256-277.

[64] A. I. Weiss, Twisted honeycombs $\{3,5,3\}_{t}$, C. R. Math. Acad. Sci. Soc. R. Can. 5 (1983), 211-215.

[65] G. Williams, Petrie schemes, Canad. J. Math. 57 (2005), 844-870.

[66] S. Wilson The smallest non-toroidal chiral maps J. Graph Theory 2 (1978), 315-318. 